\title{
A Nonlinear Least-Squares Approach for Identification of the Induction Motor Parameters
}

\author{
Kaiyu Wang, John Chiasson, Marc Bodson and Leon M. Tolbert
}

\begin{abstract}
A nonlinear least-squares method is presented for the identification of the induction motor parameters. A major difficulty with the induction motor is that the rotor state variables are not available measurements so that the system identification model cannot be made linear in the parameters without overparametrizing the model. Previous work in the literature has avoided this issue by making simplifying assumptions such as a "slowly varying speed". Here, no such simplifying assumptions are made. The problem is formulated as a nonlinear system identification problem and uses elimination theory (resultants) to compute the parameter vector that minimizes the residual error. The only assumption is that the system be sufficiently excited. The method is suitable for online operation to continuously update the parameter values. Experimental results are presented.

Index Terms - Least-Squares Identification, Induction Motor, Parameter Identification
\end{abstract}

\section{INTRODUCTION}

The induction motor parameters are the mutual inductance $M$, the stator inductance $L_{S}$, the rotor inductance $L_{R}$, the stator resistance $R_{S}$, the rotor resistance $R_{R}$, the inertia of the rotor $J$, and the load torque $\tau_{L}$. Standard methods for the estimation of the induction motor parameters include the locked rotor test, the no-load test, and the standstill frequency response test. However, these approaches cannot be used online, that is, during normal operation of the machine which is a disadvantage as some of the parameters do vary during operation. For example, field oriented control requires knowledge of the rotor time constant $T_{R}=L_{R} / R_{R}$ in order to estimate the rotor flux linkages and $R_{R}$ varies significantly due to Ohmic heating.

The work presented here is an approach to identifying the induction motor parameters based on a nonlinear leastsquares approach. As the rotor state variables are not available measurements, the system identification model cannot be made linear in the parameters without overparametrizing the model [1][2]. Previous work in the literature has avoided this issue by making simplifying assumptions such as a "slowly varying speed". Specifically, the proposed method improves upon the linear least-squares approach formulated in [1][2]. In [1][2] the approach was limited in that the acceleration was required to be small and that

K. Wang, J. Chiasson and L. M. Tolbert are with the ECE Department, The University of Tennessee, Knoxville, TN 37996. wkaiyu@utk.edu chiasson@utk.edu,tolbert@utk.edu

M. Bodson is with the EE Department, The University of Utah, Salt Lake City, UT 84112.bodson@ece.utah.edu

L. M. Tolbert is also with Oak Ridge National Laboratory, NTRC, 2360 Cherahala Boulevard, Knoxville, TN 37932.tolbertlm@ornl.gov an iterative method used to solve for the parameter vector was not guaranteed to converge nor necessarily produce the minimum residual error. Here, no such simplifying assumptions are made. The problem is formulated as a nonlinear system identification problem and uses elimination theory (resultants) to compute the parameter vector that minimizes the residual error with the only assumption being that the system is sufficiently excited. This method is suitable for online implementation so that during regular operation of the machine, the stator currents and voltages along with the rotor speed can be used to continuously update the parameters of the machine. Experimental results are presented to demonstrate the validity of the approach.

A combined parameter identification and velocity estimation problem is discussed in [3][4][5]. In contrast, we do not consider the velocity estimation problem, as the velocity is assumed to be known. On the other hand, here the velocity is allowed to vary and the paper shows how estimates of the rotor flux linkages can be simultaneously constructed (for possible use in a field-oriented control drive, such as [6]). Additionally, the method developed in this paper provides measures of the errors affecting the parameter estimates. Such measures are useful for the monitoring of the estimation procedure itself. Other related work includes [7], [8] and [9]. For background in various approaches to machine parameter estimation, there is a recent survey paper [10] and the book [11].

\section{INDUCTION MOTOR MODEL}

The work here is based on standard models of induction machines available in the literature [6]. These models neglect parasitic effects such as hysteresis, eddy currents and magnetic saturation. The particular model formulation used here is the state space model of the system given by (cf. [12][13])

$$
\begin{aligned}
\frac{d i_{S a}}{d t} & =\frac{\beta}{T_{R}} \psi_{R a}+\beta n_{p} \omega \psi_{R b}-\gamma i_{S a}+\frac{1}{\sigma L_{S}} u_{S a} \\
\frac{d i_{S b}}{d t} & =\frac{\beta}{T_{R}} \psi_{R b}-\beta n_{p} \omega \psi_{R a}-\gamma i_{S b}+\frac{1}{\sigma L_{S}} u_{S b} \\
\frac{d \psi_{R a}}{d t} & =-\frac{1}{T_{R}} \psi_{R a}-n_{p} \omega \psi_{R b}+\frac{M}{T_{R}} i_{S a} \\
\frac{d \psi_{R b}}{d t} & =-\frac{1}{T_{R}} \psi_{R b}+n_{p} \omega \psi_{R a}+\frac{M}{T_{R}} i_{S b} \\
\frac{d \omega}{d t} & =\frac{n_{p} M}{J L_{R}}\left(i_{S b} \psi_{R a}-i_{S a} \psi_{R b}\right)-\frac{\tau_{L}}{J}
\end{aligned}
$$


where $\omega=d \theta / d t$ with $\theta$ the position of the rotor, $n_{p}$ is the number of pole pairs, $i_{S a}, i_{S b}$ are the (two phase equivalent) stator currents and $\psi_{R a}, \psi_{R b}$ are the (two phase equivalent) rotor flux linkages.

As stated in the introduction, the (unknown) parameters of the model are the five electrical parameters, $R_{S}$ and $R_{R}$ (the stator and rotor resistances), $M$ (the mutual inductance), $L_{S}$ and $L_{R}$ (the stator and rotor inductances), and the two mechanical parameters, $J$ (the inertia of the rotor) and $\tau_{L}$ (the load torque). The symbols

$$
\begin{array}{ll}
T_{R}=\frac{L_{R}}{R_{R}} & \sigma=1-\frac{M^{2}}{L_{S} L_{R}} \\
\beta=\frac{M}{\sigma L_{S} L_{R}} & \gamma=\frac{R_{S}}{\sigma L_{S}}+\frac{1}{\sigma L_{S}} \frac{1}{T_{R}} \frac{M^{2}}{L_{R}}
\end{array}
$$

have been used to simplify the expressions. $T_{R}$ is referred to as the rotor time constant while $\sigma$ is called the total leakage factor.

This model is then transformed into a coordinate system attached to the rotor. For example, the current variables are transformed according to

$$
\left[\begin{array}{c}
i_{S x} \\
i_{S y}
\end{array}\right]=\left[\begin{array}{rr}
\cos \left(n_{p} \theta\right) & \sin \left(n_{p} \theta\right) \\
-\sin \left(n_{p} \theta\right) & \cos \left(n_{p} \theta\right)
\end{array}\right]\left[\begin{array}{c}
i_{S a} \\
i_{S b}
\end{array}\right] .
$$

The transformation simply projects the vectors in the $(a, b)$ frame onto the axes of the moving coordinate frame. An advantage of this transformation is that the signals in the moving frame (i.e., the $(x, y)$ frame) typically vary slower than those in the $(a, b)$ frame (they vary at the slip frequency rather than at the stator frequency). At the same time, the transformation does not depend on any unknown parameter in contrast to the field-oriented $d / q$ transformation. The stator voltages and the rotor flux linkages are transformed in the same manner as the currents resulting in the following model ([1])

$$
\begin{aligned}
\frac{d i_{S x}}{d t} & =\frac{u_{S x}}{\sigma L_{S}}-\gamma i_{S x}+\frac{\beta}{T_{R}} \psi_{R x}+n_{p} \beta \omega \psi_{R y}+n_{p} \omega i_{S y} \\
\frac{d i_{S y}}{d t} & =\frac{u_{S y}}{\sigma L_{S}}-\gamma i_{S y}+\frac{\beta}{T_{R}} \psi_{R y}-n_{p} \beta \omega \psi_{R x}-n_{p} \omega i_{S x} \\
\frac{d \psi_{R x}}{d t} & =\frac{M}{T_{R}} i_{S x}-\frac{1}{T_{R}} \psi_{R x} \\
\frac{d \psi_{R y}}{d t} & =\frac{M}{T_{R}} i_{S y}-\frac{1}{T_{R}} \psi_{R y} \\
\frac{d \omega}{d t} & =\frac{n_{p} M}{J L_{R}}\left(i_{S y} \psi_{R x}-i_{S x} \psi_{R y}\right)-\frac{\tau_{L}}{J}
\end{aligned}
$$

\section{LiNEAR OVERPARAMETRIZED MODEL}

Measurements of the stator currents $i_{S a}, i_{S b}$ and voltages $u_{S a}, u_{S b}$ as well as the position $\theta$ of the rotor are assumed to be available (velocity may then be reconstructed from position measurements). However, the rotor flux linkages $\psi_{R x}, \psi_{R y}$ are not assumed to be measured. Standard methods for parameter estimation are based on equalities where known signals depend linearly on unknown parameters. However, the induction motor model described above does not fit in this category unless the rotor flux linkages are measured. The first step is to eliminate the fluxes $\psi_{R x}, \psi_{R y}$ and their derivatives $d \psi_{R x} / d t, d \psi_{R y} / d t$. The four equations (3), (4), (5), (6) can be used to solve for $\psi_{R x}, \psi_{R y}, d \psi_{R x} / d t, d \psi_{R y} / d t$, but one is left without another independent equation(s) to set up a regressor system for the identification algorithm. A new set of independent equations are found by differentiating equations (3) and (4) to obtain

$$
\begin{aligned}
\frac{1}{\sigma L_{s}} \frac{d u_{S x}}{d t} & =\frac{d^{2} i_{S x}}{d t^{2}}+\gamma \frac{d i_{S x}}{d t}-\frac{\beta}{T_{R}} \frac{d \psi_{R x}}{d t}-n_{p} \beta \omega \frac{d \psi_{R y}}{d t} \\
& -n_{p} \beta \psi_{R y} \frac{d \omega}{d t}-n_{p} \omega \frac{d i_{S y}}{d t}-n_{p} i_{S y} \frac{d \omega}{d t}
\end{aligned}
$$

$$
\begin{aligned}
\frac{1}{\sigma L_{s}} \frac{d u_{S y}}{d t} & =\frac{d^{2} i_{S y}}{d t^{2}}+\gamma \frac{d i_{S y}}{d t}-\frac{\beta}{T_{R}} \frac{d \psi_{R y}}{d t}+n_{p} \beta \omega \frac{d \psi_{R x}}{d t} \\
& +n_{p} \beta \psi_{R x} \frac{d \omega}{d t}+n_{p} \omega \frac{d i_{S x}}{d t}+n_{p} i_{S x} \frac{d \omega}{d t} .
\end{aligned}
$$

Next, equations (3), (4), (5), (6) are solved for $\psi_{R x}, \psi_{R y}$, $d \psi_{R x} / d t, d \psi_{R y} / d t$ and substituted into equations (8) and (9) to obtain

$$
\begin{aligned}
0 & =-\frac{d^{2} i_{S x}}{d t^{2}}+\frac{d i_{S y}}{d t} n_{p} \omega+\frac{1}{\sigma L_{S}} \frac{d u_{S x}}{d t}-\left(\gamma+\frac{1}{T_{R}}\right) \frac{d i_{S x}}{d t} \\
& -i_{S x}\left(-\frac{\beta M}{T_{R}^{2}}+\frac{\gamma}{T_{R}}\right)+i_{S y} n_{p} \omega\left(\frac{1}{T_{R}}+\frac{\beta M}{T_{R}}\right) \\
& +\frac{u_{S x}}{\sigma L_{S} T_{R}}+n_{p} \frac{d \omega}{d t} i_{S y}-n_{p} \frac{d \omega}{d t} \frac{1}{\sigma L_{S}\left(1+n_{p}^{2} \omega^{2} T_{R}^{2}\right)} \times \\
& \left(-\sigma L_{S} T_{R} \frac{d i_{S y}}{d t}-\gamma i_{S y} \sigma L_{S} T_{R}-i_{S x} n_{p} \omega \sigma L_{S} T_{R}\right. \\
& -\frac{d i_{S x}}{d t} n_{p} \omega \sigma L_{S} T_{R}^{2}-\gamma i_{S x} n_{p} \omega \sigma L_{S} T_{R}^{2}+ \\
& \left.i_{S y} n_{p}^{2} \omega^{2} \sigma L_{S} T_{R}^{2}+n_{p} \omega T_{R}^{2} u_{S x}+T_{R} u_{S y}\right)
\end{aligned}
$$

and

$$
\begin{aligned}
0 & =-\frac{d^{2} i_{S y}}{d t^{2}}-\frac{d i_{S x}}{d t} n_{p} \omega+\frac{1}{\sigma L_{S}} \frac{d u_{S y}}{d t}-\left(\gamma+\frac{1}{T_{R}}\right) \frac{d i_{S y}}{d t} \\
& -i_{S y}\left(-\frac{\beta M}{T_{R}^{2}}+\frac{\gamma}{T_{R}}\right)-i_{S x} n_{p} \omega\left(\frac{1}{T_{R}}+\frac{\beta M}{T_{R}}\right) \\
& +\frac{u_{S y}}{\sigma L_{S} T_{R}}-n_{p} \frac{d \omega}{d t} i_{S x}+n_{p} \frac{d \omega}{d t} \frac{1}{\sigma L_{S}\left(1+n_{p}^{2} \omega^{2} T_{R}^{2}\right)} \times \\
& \left(-\sigma L_{S} T_{R} \frac{d i_{S x}}{d t}-\gamma i_{S x} \sigma L_{S} T_{R}+i_{S y} n_{p} \omega \sigma L_{S} T_{R}\right. \\
& +\frac{d i_{S y}}{d t} n_{p} \omega \sigma L_{S} T_{R}^{2}+\gamma i_{S y} n_{p} \omega \sigma L_{S} T_{R}^{2} \\
& \left.+i_{S x} n_{p}^{2} \omega^{2} \sigma L_{S} T_{R}^{2}-n_{p} \omega T_{R}^{2} u_{S y}+T_{R} u_{S x}\right) .
\end{aligned}
$$

The set of equations (10) and (11) may be rewritten in regressor form as

$$
y(t)=W(t) K
$$


where $K \in R^{15}, W \in R^{2 \times 15}$ and $y \in R^{2}$ are given by

$$
\begin{aligned}
& K \triangleq\left[\begin{array}{lllllll}
\gamma & \beta M & \frac{1}{\sigma L_{S}} & \frac{\beta M}{T_{R}^{2}} & \frac{1}{T_{R}} & \frac{\gamma}{T_{R}} & \frac{\beta M}{T_{R}}
\end{array} \quad T_{R}\right. \\
& \left.\begin{array}{ccccccc}
\gamma T_{R} & \beta M T_{R} & T_{R}^{2} & \gamma T_{R}^{2} & \frac{T_{R}^{2}}{\sigma L_{S}} & \frac{1}{\sigma L_{S} T_{R}} & \frac{T_{R}}{\sigma L_{S}}
\end{array}\right]^{T}
\end{aligned}
$$

$W(t) \triangleq$

$$
\begin{aligned}
& {\left[\begin{array}{ccccc}
-\frac{d i_{S x}}{d t} & n_{p}^{2} \omega^{2} i_{S x} & \frac{d u_{S x}}{d t} & i_{S x} & n_{p} \omega i_{S y}-\frac{d i_{S x}}{d t} \\
-\frac{d i_{S y}}{d t} & n_{p}^{2} \omega^{2} i_{S y} & \frac{d u_{S y}}{d t} & i_{S y} & -n_{p} \omega i_{S x}-\frac{d i_{S y}}{d t}
\end{array}\right.} \\
& -i_{S x} \quad n_{p} \omega i_{S y} \quad-n_{p}^{2} \omega^{2} \frac{d i_{S x}}{d t}+n_{p}^{3} \omega^{3} i_{S y}+ \\
& -i_{S y} \quad-n_{p} \omega i_{S x} \quad-n_{p}^{2} \omega^{2} \frac{d i_{S y}}{d t}-n_{p}^{3} \omega^{3} i_{S x}+ \\
& \frac{d \omega}{d t}\left(n_{p} \frac{d i_{S y}}{d t}+n_{p}^{2} \omega i_{S x}\right) \quad n_{p} i_{S y} \frac{d \omega}{d t}-n_{p}^{2} \omega^{2} i_{S x} \\
& \frac{d \omega}{d t}\left(-n_{p} \frac{d i_{S x}}{d t}+n_{p}^{2} \omega i_{S y}\right) \quad-n_{p} i_{S x} \frac{d \omega}{d t}-n_{p}^{2} \omega^{2} i_{S y} \\
& n_{p}^{3} \omega^{3} i_{S y} \quad n_{p}^{2}\left(\omega \frac{d i_{S x}}{d t} \frac{d \omega}{d t}-\omega^{2} \frac{d^{2} i_{S x}}{d t^{2}}\right)+n_{p}^{3} \omega^{3} \frac{d i_{S y}}{d t} \\
& -n_{p}^{3} \omega^{3} i_{S x} \quad n_{p}^{2}\left(\omega \frac{d i_{S y}}{d t} \frac{d \omega}{d t}-\omega^{2} \frac{d^{2} i_{S y}}{d t^{2}}\right)-n_{p}^{3} \omega^{3} \frac{d i_{S x}}{d t} \\
& n_{p}^{2}\left(\omega i_{S x} \frac{d \omega}{d t}-\omega^{2} \frac{d i_{S x}}{d t}\right) \quad n_{p}^{2}\left(\omega^{2} \frac{d u_{S x}}{d t}-u_{S x} \omega \frac{d \omega}{d t}\right) \\
& n_{p}^{2}\left(\omega i_{S y} \frac{d \omega}{d t}-\omega^{2} \frac{d i_{S y}}{d t}\right) \quad n_{p}^{2}\left(\omega^{2} \frac{d u_{S y}}{d t}-u_{S y} \omega \frac{d \omega}{d t}\right) \\
& u_{S x} \quad n_{p}^{2} \omega^{2} u_{S x}-n_{p} u_{S y} \frac{d \omega}{d t} \\
& u_{S y} \quad n_{p}^{2} \omega^{2} u_{S y}+n_{p} u_{S x} \frac{d \omega}{d t}
\end{aligned}
$$

and

$$
y(t) \triangleq\left[\begin{array}{c}
\frac{d^{2} i_{S x}}{d t^{2}}-n_{p} i_{S y} \frac{d \omega}{d t}-n_{p} \omega \frac{d i_{S y}}{d t} \\
\frac{d^{2} i_{S y}}{d t^{2}}+n_{p} i_{S x} \frac{d \omega}{d t}+n_{p} \omega \frac{d i_{S x}}{d t}
\end{array}\right] .
$$

Though the system (12) is linear in the parameters, it is overparameterized resulting in poor numerical conditioning if standard least-squares techniques are used. Specifically,

$$
\begin{aligned}
K_{1} & =K_{6} K_{8}, K_{2}=K_{4} K_{8}^{2}, K_{3}=K_{8} K_{14}, K_{5}=1 / K_{8}, \\
K_{7} & =K_{4} K_{8}, K_{9}=K_{6} K_{8}^{2}, K_{10}=K_{4} K_{8}^{3}, K_{11}=K_{8}^{2}, \\
K_{12} & =K_{6} K_{8}^{3}, K_{13}=K_{14} K_{8}^{3}, K_{15}=K_{14} K_{8}^{2}
\end{aligned}
$$

so that only the four parameters $K_{4}, K_{6}, K_{8}, K_{14}$ are independent. Also, not all five electrical parameters $R_{S}$, $L_{S}, R_{R}, L_{R}$ and $M$ can be retrieved from the $K_{i}$ 's. The four parameters $K_{4}, K_{6}, K_{8}, K_{14}$ determine only the four independent parameters $R_{S}, L_{S}, \sigma$ and $T_{R}$ by

$R_{S}=\frac{K_{6}-K_{4}}{K_{14}}, T_{R}=K_{8}, L_{S}=\frac{1+K_{4} K_{8}^{2}}{K_{14} K_{8}}, \sigma=\frac{1}{1+K_{4} K_{8}^{2}}$.

As $T_{R}=L_{R} / R_{R}$ and $\sigma=1-M^{2} /\left(L_{S} L_{R}\right)$, only $L_{R} / R_{R}$ and $M^{2} / L_{R}$ can be obtained, and not $M, L_{R}$, and $R_{R}$ independently. This situation is inherent to the identification problem when rotor flux linkages are unknown and is not specific to the method. If the rotor flux linkages are not measured, machines with different $R_{R}, L_{R}$, and $M$, but identical $L_{R} / R_{R}$ and $M^{2} / L_{R}$ will have the same input/output (i.e., voltage to current and speed) characteristics. Specifically, the transformation ratio from stator to rotor cannot be determined unless rotor measurements are taken. However, machines with different $K_{i}$ parameters, yet satisfying the nonlinear relationships (13), will be distinguishable. For a related discussion of this issue, see Bellini et al [14] where parameter identification is performed using torque-speed and stator current-speed characteristics.

\section{NONLINEAR LEAST-SQUARES IDENTIFICATION[15][16]}

In this section, it is shown how the linear least-squares method is modified to account for the constraint (13). The problem is to minimize

$$
\begin{aligned}
E^{2}(K) & =\sum_{n=1}^{N}|y(n)-W(n) K|^{2} \\
& =R_{y}-2 R_{W y}^{T} K+K^{T} R_{W} K
\end{aligned}
$$

subject to the constraints (13). On physical grounds, the parameters $K_{4}, K_{6}, K_{8}, K_{14}$ are constrained to

$$
0<K_{i}<\infty \text { for } i=4,6,8,14 \text {. }
$$

Also, based on physical grounds, the squared error $E^{2}(K)$ will be minimized in the interior of this region. Let

$$
\begin{gathered}
E^{2}\left(K_{p}\right) \triangleq \sum_{n=1}^{N}|y(n)-W(n) K|_{\substack{K_{1}=K_{6} K_{8} \\
K_{2}=K_{4} K_{8}^{2}}}^{2} \\
\vdots \\
=R_{y}-\left.2 R_{W y}^{T} K\right|_{\substack{K_{1}=K_{6} K_{8} \\
K_{2}=K_{4} K_{8}^{2}}}+\left.\left(K^{T} R_{W} K\right)\right|_{\substack{K_{1}=K_{6} K_{8} \\
K_{2}=K_{4} K_{8}^{2}}}
\end{gathered}
$$

where

$$
K_{p} \triangleq\left[\begin{array}{llll}
K_{4} & K_{6} & K_{8} & K_{14}
\end{array}\right]^{T} .
$$

As just explained, the minimum of (17) must occur in the interior of the region and therefore at an extremum point. This then entails solving the four equations

$$
\begin{aligned}
& r_{1}\left(K_{p}\right) \triangleq \frac{\partial E^{2}\left(K_{p}\right)}{\partial K_{4}}=0 \\
& r_{2}\left(K_{p}\right) \triangleq \frac{\partial E^{2}\left(K_{p}\right)}{\partial K_{6}}=0 \\
& r_{3}\left(K_{p}\right) \triangleq \frac{\partial E^{2}\left(K_{p}\right)}{\partial K_{8}}=0 \\
& r_{4}\left(K_{p}\right) \triangleq \frac{\partial E^{2}\left(K_{p}\right)}{\partial K_{14}}=0 .
\end{aligned}
$$


The partial derivatives in (18)-(21) are rational functions in the parameters $K_{4}, K_{6}, K_{8}, K_{14}$. Defining

$$
\begin{aligned}
& p_{1}\left(K_{p}\right) \triangleq K_{8} r_{1}\left(K_{p}\right)=K_{8} \frac{\partial E^{2}\left(K_{p}\right)}{\partial K_{4}} \\
& p_{2}\left(K_{p}\right) \triangleq K_{8} r_{2}\left(K_{p}\right)=K_{8} \frac{\partial E^{2}\left(K_{p}\right)}{\partial K_{6}} \\
& p_{3}\left(K_{p}\right) \triangleq K_{8}^{3} r_{3}\left(K_{p}\right)=K_{8}^{3} \frac{\partial E^{2}\left(K_{p}\right)}{\partial K_{8}} \\
& p_{4}\left(K_{p}\right) \triangleq K_{8} r_{4}\left(K_{p}\right)=K_{8} \frac{\partial E^{2}\left(K_{p}\right)}{\partial K_{14}}
\end{aligned}
$$

results in the $p_{i}\left(K_{p}\right)$ being polynomials in the parameters $K_{4}, K_{6}, K_{8}, K_{14}$ and having the same positive zero set (i.e., the same roots satisfying $K_{i}>0$ ) as the system (18)-(21). The degrees of the polynomials $p_{i}$ are given in the table below.

\begin{tabular}{|c|c|c|c|c|}
\hline & $\operatorname{deg} K_{4}$ & $\operatorname{deg} K_{6}$ & $\operatorname{deg} K_{8}$ & $\operatorname{deg} K_{14}$ \\
\hline$p_{1}\left(K_{p}\right)$ & 1 & 1 & 7 & 1 \\
\hline$p_{2}\left(K_{p}\right)$ & 1 & 1 & 7 & 1 \\
\hline$p_{3}\left(K_{p}\right)$ & 2 & 2 & 8 & 2 \\
\hline$p_{4}\left(K_{p}\right)$ & 1 & 1 & 7 & 1 \\
\hline
\end{tabular}

All possible solutions to this set may be found using elimination theory as is now summarized.

\section{A. Solving Systems of Polynomial Equations [17][18]}

The question at hand is "Given two polynomial equations $a\left(K_{1}, K_{2}\right)=0$ and $b\left(K_{1}, K_{2}\right)=0$, how does one solve them simultaneously to eliminate (say) $K_{2}$ ?". A systematic procedure to do this is known as elimination theory and uses the notion of resultants. Briefly, one considers $a\left(K_{1}, K_{2}\right)$ and $b\left(K_{1}, K_{2}\right)$ as polynomials in $K_{2}$ whose coefficients are polynomials in $K_{1}$. Then, for example, letting $a\left(K_{1}, K_{2}\right)$ and $b\left(K_{1}, K_{2}\right)$ have degrees 3 and 2 , respectively in $K_{2}$, they may be written in the form

$$
\begin{aligned}
a\left(K_{1}, K_{2}\right)= & a_{3}\left(K_{1}\right) K_{2}^{3}+a_{2}\left(K_{1}\right) K_{2}^{2}+a_{1}\left(K_{1}\right) K_{2} \\
& +a_{0}\left(K_{1}\right) \\
b\left(K_{1}, K_{2}\right)= & b_{2}\left(K_{1}\right) K_{2}^{2}+b_{1}\left(K_{1}\right) K_{2}+b_{0}\left(K_{1}\right) .
\end{aligned}
$$

The $n \times n$ Sylvester matrix, where $n=$ $\operatorname{deg}_{K_{2}}\left\{a\left(K_{1}, K_{2}\right)\right\}+\operatorname{deg}_{K_{2}}\left\{b\left(K_{1}, K_{2}\right)\right\}=3+2=5$, is defined by $S_{a, b}\left(K_{1}\right) \triangleq$

$$
\left[\begin{array}{ccccc}
a_{0}\left(K_{1}\right) & 0 & b_{0}\left(K_{1}\right) & 0 & 0 \\
a_{1}\left(K_{1}\right) & a_{0}\left(K_{1}\right) & b_{1}\left(K_{1}\right) & b_{0}\left(K_{1}\right) & 0 \\
a_{2}\left(K_{1}\right) & a_{1}\left(K_{1}\right) & b_{2}\left(K_{1}\right) & b_{1}\left(K_{1}\right) & b_{0}\left(K_{1}\right) \\
a_{3}\left(K_{1}\right) & a_{2}\left(K_{1}\right) & 0 & b_{2}\left(K_{1}\right) & b_{1}\left(K_{1}\right) \\
0 & a_{3}\left(K_{1}\right) & 0 & 0 & b_{2}\left(K_{1}\right)
\end{array}\right] .
$$

The resultant polynomial is then defined by

$r\left(K_{1}\right)=\operatorname{Res}\left(a\left(K_{1}, K_{2}\right), b\left(K_{1}, K_{2}\right), K_{2}\right) \triangleq \operatorname{det} S_{a, b}\left(K_{1}\right)$

and is the result of eliminating the variable $K_{2}$ from $a\left(K_{1}, K_{2}\right)$ and $b\left(K_{1}, K_{2}\right)$. In fact, the following is true.

Theorem 1: [17][18] Any solution $\left(K_{10}, K_{20}\right)$ of $a\left(K_{1}, K_{2}\right)=0$ and $b\left(K_{1}, K_{2}\right)=0$ must have $r\left(K_{10}\right)=0$.
Though the converse of this theorem is not necessarily true, the finite number of solutions of $r\left(K_{1}\right)=0$ are the only possible candidates for the first coordinate (partial solutions) of the common zeros of $a\left(K_{1}, K_{2}\right)$ and $b\left(K_{1}, K_{2}\right)$. Whether or not such a partial solution extends to a full solution is easily determined by back solving and checking the solution.

Using the polynomials (22)-(25) and the computer algebra software program MATHEMATICA [19], the variable $K_{4}$ is eliminated first to obtain three polynomials in three unknowns as

$$
\begin{aligned}
& r_{p 1 p 2}\left(K_{6}, K_{8}, K_{14}\right) \triangleq \operatorname{Res}\left(p_{1}, p_{2}, K_{4}\right) \\
& r_{p 1 p 3}\left(K_{6}, K_{8}, K_{14}\right) \triangleq \operatorname{Res}\left(p_{1}, p_{2}, K_{4}\right) \\
& \left.r_{p 1 p 4}\left(K_{6}, K_{8}, K_{14}\right) \triangleq \operatorname{Res}\left(p_{1}, p_{2}\right), K_{4}\right)
\end{aligned}
$$

where

\begin{tabular}{|c|c|c|c|}
\hline & $\operatorname{deg} K_{6}$ & $\operatorname{deg} K_{8}$ & $\operatorname{deg} K_{14}$ \\
\hline$r_{p 1 p 2}$ & 1 & 14 & 1 \\
\hline$r_{p 1 p 3}$ & 2 & 22 & 2 \\
\hline$r_{p 1 p 4}$ & 1 & 14 & 1 \\
\hline
\end{tabular}

Next $K_{6}$ is eliminated to obtain two polynomials in two unknowns as

$$
\begin{aligned}
& r_{p 1 p 2 p 3}\left(K_{8}, K_{14}\right) \triangleq \operatorname{Res}\left(r_{p 1 p 2}, r_{p 1 p 3}, K_{6}\right) \\
& r_{p 1 p 2 p 4}\left(K_{8}, K_{14}\right) \triangleq \operatorname{Res}\left(r_{p 1 p 2}, r_{p 1 p 4}, K_{6}\right)
\end{aligned}
$$

where

\begin{tabular}{|c|c|c|}
\hline & $\operatorname{deg} K_{8}$ & $\operatorname{deg} K_{14}$ \\
\hline$r_{p 1 p 2 p 3}$ & 50 & 2 \\
\hline$r_{p 1 p 2 p 4}$ & 28 & 1 \\
\hline
\end{tabular}

Finally $K_{14}$ is eliminated to obtain a single polynomial in $K_{8}$ as

$r\left(K_{8}\right) \triangleq \operatorname{Res}\left(r_{p 1 p 2 p 3}\left(K_{8}, K_{14}\right), r_{p 1 p 2 p 4}\left(K_{8}, K_{14}\right), K_{14}\right)$

where

$$
\operatorname{deg} K_{8}=104
$$

The parameter $K_{8}$ was chosen as the variable not eliminated because its degree was the highest at each step meaning it would have a larger (in dimension) Sylvester matrix than using any other variable. The positive roots of $r\left(K_{8}\right)=0$ are found which are then substituted into $r_{p 1 p 2 p 3}=0$ (or $r_{p 1 p 2 p 4}=0$ ) which in turn are solved to obtain the partial solutions $\left(K_{8}, K_{14}\right)$. The partial solutions $\left(K_{8}, K_{14}\right)$ are then substituted into $r_{p 1 p 2}=0$ (or $r_{p 1 p 3}=0$ or $r_{p 1 p 4}=0$ ) which are solved to obtain the partial solutions $\left(K_{6}, K_{8}, K_{14}\right)$ so that they in turn may be substituted into $p_{1}=0$ (or $p_{2}=0$ or $p_{3}=0$ or $p_{4}=0$ ) which are solved to obtain the solutions $\left(K_{4}, K_{6}, K_{8}, K_{14}\right)$. These solutions are then checked to see which ones satisfy the complete system of polynomials equations (22)-(25) and those that do constitute the candidate solutions for the minimization. Based on physical considerations, the set of 
candidate solutions is non empty. From the set of candidate solutions, the one that gives the smallest squared error is chosen.

\section{B. Mechanical Parameters}

Once the electrical parameters have been found, the two mechanical parameters $J, f\left(\tau_{L}=-f \omega\right)$ can be found using a linear least-squares algorithm. To do so, equations (8) and (9) are solved for $M \psi_{R x} / L_{R}, M \psi_{R y} / L_{R}$ resulting in

$$
\begin{aligned}
& {\left[\begin{array}{l}
M \psi_{R x} / L_{R} \\
M \psi_{R y} / L_{R}
\end{array}\right]=\frac{\sigma L_{S}}{\left(1 / T_{R}\right)^{2}+n_{p}^{2} \omega^{2}}\left[\begin{array}{cc}
1 / T_{R} & -\omega \\
\omega & 1 / T_{R}
\end{array}\right] \times} \\
& {\left[\begin{array}{l}
d i_{S x} / d t-u_{S x} /\left(\sigma L_{S}\right)+\gamma i_{S x}-n_{p} \omega i_{S y} \\
d i_{S y} / d t-u_{S y} /\left(\sigma L_{S}\right)+\gamma i_{S y}+n_{p} \omega i_{S x}
\end{array}\right] .}
\end{aligned}
$$

Noting that

$$
\gamma=\frac{R_{S}}{\sigma L_{S}}+\frac{1}{\sigma L_{S}} \frac{1}{T_{R}} \frac{M^{2}}{L_{R}}=\frac{R_{S}}{\sigma L_{S}}+\frac{1}{\sigma L_{S}} \frac{1}{T_{R}}(1-\sigma) L_{S},
$$

then quantities on the right hand side of (27) are all known once the electrical parameters have been computed. With $K_{16} \triangleq n_{p} / J, K_{17} \triangleq f / J$, equation (7) may be rewritten as

$$
\frac{d \omega}{d t}=\left[\frac{M \psi_{R x}}{L_{R}} i_{S y}-\frac{M \psi_{R y}}{L_{R}} i_{S x} \quad-\omega\right]\left[\begin{array}{c}
K_{16} \\
K_{17}
\end{array}\right]
$$

so that the standard linear squares approach is directly applicable. Then

$$
J \triangleq n_{p} / K_{16}, f \triangleq n_{p} K_{17} / K_{16} .
$$

\section{EXPERIMENTAL RESULTS}

A three phase, $0.5 \mathrm{hp}, 1735 \mathrm{rpm}\left(n_{p}=2\right.$ pole-pair $)$ induction machine was used for the experiments. A 4096 pulse/rev optical encoder was attached to the motor for position measurements. The motor was connected to a threephase, $60 \mathrm{~Hz}, 230 \mathrm{~V}$ source through a switch with no load on the machine. When the switch was closed, the stator currents and voltages along with the rotor position were sampled at $4 \mathrm{kHz}$. Filtered differentiation (using digital filters) was used for calculating the acceleration and the derivatives of the voltages and currents. Specifically, the signals were filtered with a lowpass digital Butterworth filter followed by reconstruction of the derivatives using $d x(t) / d t=(x(t)-x(t-T)) / T$ where $T$ is the sampling interval. The voltages and currents were put through a $3-2$ transformation to obtain the two phase equivalent voltages $u_{S a}, u_{S b}$. The sampled two phase equivalent current $i_{S a}$ and its simulated response $i_{S a_{-} \text {sim }}$ are shown in Figure 1 (The simulated current will be discussed below). The phase $b$ current $i_{S b}$ is similar, but shifted by $\pi /\left(2 n_{p}\right)$. The calculated speed $\omega$ (from the position measurements) and the simulated speed $\omega_{\text {sim }}$ are shown in Figure 2 (the simulated speed $\omega_{\text {sim }}$ will be discussed below). Using the data $\left\{u_{S a}, u_{S b}, i_{S a}, i_{S b}, \theta\right\}$ collected between $5.57 \mathrm{sec}$ to $5.8 \mathrm{sec}$, the quantities $u_{S x}, u_{S y}, d u_{S x} / d t, d u_{S y} / d t$,

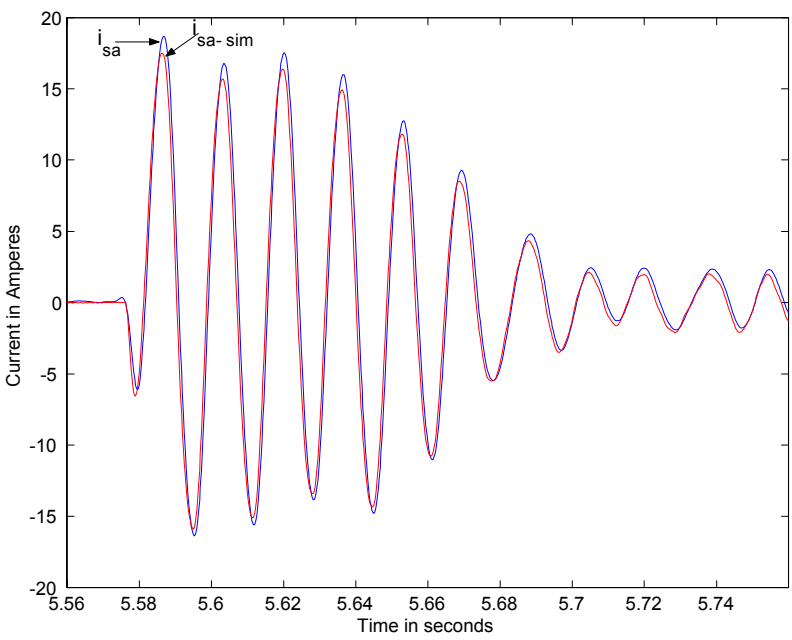

Fig. 1. Phase $a$ current $i_{S a}$ and its simulated response $i_{S a_{-} \text {sim. }}$

$i_{S x}, i_{S y} d i_{S x} / d t, d i_{S y} / d t, d^{2} i_{S x} / d t^{2}, d^{2} i_{S y} / d t^{2}, \omega=$ $d \theta / d t, d \omega / d t$ were calculated and the regressor matrices $R_{W}, R_{y}$ and $R_{W y}$ were computed. The procedure explained in Section IV was then carried out to compute $K_{4}, K_{6}, K_{8}, K_{14}$. In this case, there was only one extremum point that had positive values for all the $K_{i}$. The table below presents the parameter values determined using the nonlinear least-squares methodology along with their corresponding parametric error indices [2].

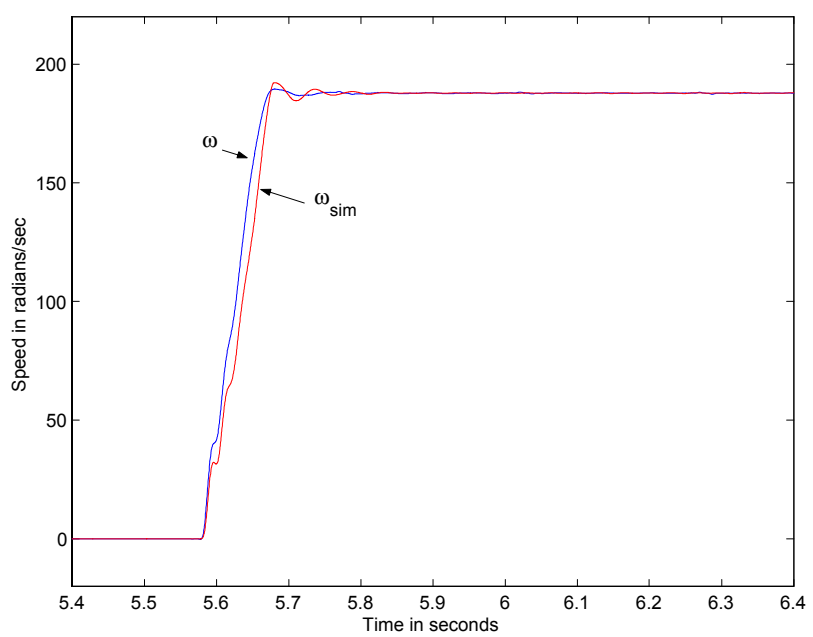

Fig. 2. Calculated speed $\omega$ and simulated speed $\omega_{\text {sim }}$.

\begin{tabular}{|c|c|c|}
\hline Parameter & Estimated Value & Parametric Error \\
\hline$K 4$ & 519.7 & 185.8 \\
\hline$K 6$ & 1848.3 & 796.4 \\
\hline$K 8$ & 0.1311 & 0.0103 \\
\hline$K 14$ & 259.5 & 59.4 \\
\hline
\end{tabular}

The residual error index was calculated to be $13.43 \%$ [2].

The motor's electrical parameters are computed using (14) 
to obtain

$$
\begin{aligned}
& R_{S}=5.12 \mathrm{Ohms}, T_{R}=0.1311 \mathrm{sec} \\
& L_{S}=0.2919 \mathrm{H}, \sigma=0.1007
\end{aligned}
$$

By way of comparison, the stator resistance was measured using an Ohmmeter giving the value of $4.9 \mathrm{Ohms}$, and a no load test was also run to compute the value of $L_{S}$ resulting in $0.33 \mathrm{H}$.

Using the electrical parameters, the rotor flux linkages $\left(M / L_{R}\right) \psi_{R x}$ and $\left(M / L_{R}\right) \psi_{R y}$ were reconstructed and used to identify the mechanical parameters. The table below gives the estimated values and the parametric error indices.

\begin{tabular}{|c|c|c|}
\hline Parameter & Estimated Value & Parametric Error \\
\hline$K_{16}$ & 952.38 & 126.92 \\
\hline$K_{17}$ & 0.5714 & 0.1528 \\
\hline
\end{tabular}

The residual error index was calculated to be $18.6 \%$. The $2 \times 2$ regressor matrix $R_{W}$ for these two parameters had a condition number of $1.060 \times 10^{3}$ [20]. The corresponding values for the motor parameters $J$ and $f$ are then computed using (29) to obtain

$$
\begin{aligned}
J & =n_{p} / K_{16}=0.0021 \mathrm{kgm}^{2} \\
f & =n_{p} K_{17} / K_{16}=0.0012 \mathrm{Nm} /(\mathrm{rad} / \mathrm{sec}) .
\end{aligned}
$$

\section{A. Simulation of the Experimental Motor}

Another useful way to evaluate the identified parameters (30)-(31) and (32)-(33) is to simulate the motor using these values with the measured voltages as input. One then compares the simulation's output (stator currents) with the measured outputs. The experimental voltages were then used as input to a simulation of the model using the parameter values from (30)-(31) and (32)-(33). The resulting phase $a$ current $i_{S a_{-} \text {sim }}$ from the simulation is shown in Figure 1 and corresponds well with the actual measured current $i_{S a}$. Similarly, the resulting speed $\omega_{\text {sim }}$ from the simulation is shown in Figure 2.

\section{DISCUSSION}

The technique proposed here is relevant for other systems and not just for induction machine parameter identification. The first issue of concern in applying this methodology to other systems is that the parameters used to develop a linear overparameterized model be rationally related. The next issue is the symbolic computation of the Sylvester matrices to compute the resultant polynomials. As the degrees of the polynomials to be solved increase, the dimension of the corresponding Sylvester matrices increase, and therefore the symbolic computation of their determinants becomes more computationally intensive. The particular application considered in this work did not experience such computational difficulty. Interestingly, the recent work of [21][22] is promising for the efficient symbolic computation of the determinants of large Sylvester matrices.

\section{ACKNOWLEDGEMENTS}

Drs. Chiasson and Tolbert would like to thank Oak Ridge National Laboratory for partially supporting this work through the UT/Battelle contract no. 4000023754. Dr. Tolbert thanks the National Science Foundation for partially supporting this work through contract NSF ECS-0093884.

\section{REFERENCES}

[1] J. Stephan, "Real-time estimation of the parameters and fluxes of induction motors," Master's thesis, Carnegie Mellon University, 1992.

[2] J. Stephan, M. Bodson, and J. Chiasson, "Real-time estimation of induction motor parameters," IEEE Transactions on Industry Applications, vol. 30, pp. 746-759, May/June 1994.

[3] M. Vélez-Reyes, W. L. Fung, and J. E. Ramos-Torres, "Developing robust algorithms for speed and parameter estimation in induction machines," in Proceedings of the IEEE Conference on Decision and Control, 2001. Orlando Florida.

[4] M. Vélez-Reyes, K. Minami, and G. Verghese, "Recursive speed and parameter estimation for induction machines," in Proceedings of the IEEE Industry Applications Conference, 1989. San Diego, California.

[5] M. Vélez-Reyes and G. Verghese, "Decomposed algorithms for speed and parameter estimation in induction machines," in Proceedings of the IFAC Nonlinear Control Systems Design Symposium, pp. 156161, 1992. Bordeaux, France.

[6] W. Leonhard, Control of Electrical Drives, 2nd Edition. SpringerVerlag, 1997.

[7] M. Cirrincione, M. Pucci, G. Cirrincione, and G.-A. Capolino, "A new experimental application of least-squares techniques for the estimation of the induction motor parameters," IEEE Transactions on Industry Applications, vol. 39, pp. 1247-1256, September/October 2003.

[8] S. R. Shaw and S. B. Leeb, "Identification of induction motor parameters from transient stator current measurements," IEEE Transactions on Industrial Electronics, vol. 46, pp. 139-149, February 1999.

[9] M. Vélez-Reyes, M. Mijalkovic, A. M. Stankovic, S. Hiti, and J. Nagashima, "Output selection for tuning of field oriented controllers: Steady state analysis," in Conference Record of Industry Applications Society, pp. 2012-2016, October 2003. Salt Lake City UT.

[10] H. A. Toliyat, E. Levi, and M. Raina, "A review of RFO induction motor parameter estimation techniques," IEEE Transactions on Energy Conversion, vol. 18, pp. 271-283, June 2003.

[11] P. Vas, Parameter estimation, condition monitoring, and diagnosis of electrical machines. Oxford: Clarendon Press, 1993.

[12] M. Bodson, J. Chiasson, and R. Novotnak, "High performance induction motor control via input-output linearization," IEEE Control Systems Magazine, vol. 14, pp. 25-33, August 1994.

[13] R. Marino, S. Peresada, and P. Valigi, "Adaptive input-output linearizing control of induction motors," IEEE Transactions on Automatic Control, vol. 38, pp. 208-221, February 1993.

[14] A. Bellini, A. D. Carli, and M. L. Cava, "Parameter identification for induction motor simulation," Automatica, vol. 12, pp. 383-386, 1976.

[15] L. Ljung, System Identification: Theory for the User. Prentice-Hall Inc., 1986.

[16] Shankar Sastry and Marc Bodson, Adaptive Control: Stability, Convergence, and Robustness. Prentice Hall, 1989.

[17] D. Cox, J. Little, and D. O'Shea, IDEALS, VARIETIES, AND ALGORITHMS An Introduction to Computational Algebraic Geometry and Commutative Algebra, Second Edition. Springer-Verlag, 1996.

[18] Joachim von zur Gathen and Jürgen Gerhard, Modern Computer Algebra. Cambridge University Press, 1999.

[19] S. Wolfram, Mathematica, A System for Doing Mathematics by Computer, Second Edition. Addison-Wesley, 1992.

[20] D. Kincaid and W. Cheney, Numerical Analysis: Mathematics of Scientific Computing. Brooks/Cole, 2002.

[21] M. Hromcik and M. Sebek, "New algorithm for polynomial matrix determinant based on FFT," in Proceedings of the European Conference on Control ECC'99, August 1999. Karlsruhe Germany.

[22] M. Hromcik and M. Sebek, "Numerical and symbolic computation of polynomial matrix determinant," in Proceedings of the 1999 Conference on Decision and Control, 1999. Tampa FL. 